\title{
CONTROLE DOS VASOS RENAIS USANDO CLIPS VASCULARES E FIO CIRÚRGICO EM NEFRECTOMIAS VÍDEO-ASSISTIDAS DE DOADORES VIVOS
}

\author{
CONTROL OF RENAL VESSELS USING TITANIUM CLIPS AND COTTON SUTURE IN \\ HAND-ASSISTED LAPAROSCOPIC LIVE DONOR NEPHRECTOMY
}

\author{
Alcides José Branco Filho, ACBC-PR'; ; Anibal Wood Branco²; William Kondo,AsCBC-PR ; \\ Rafael Fabio Maciel $^{4}$; Ronaldo Moreno de Carvalho ${ }^{5}$; Mariana Jorge Garcia ${ }^{6}$
}

\begin{abstract}
RESUMO: Objetivo: A nefrectomia laparoscópica em doadores vivos para transplante renal vem assumindo um papel importante na era das cirurgias minimamente invasivas, acarretando menor morbidade aos doadores, e resultados semelhantes à técnica aberta no que se refere ao enxerto renal. O objetivo do presente artigo é relatar a experiência do nosso serviço utilizando a técnica de controle dos vasos renais usando fio cirúrgico e clips vasculares. Método: Foram realizadas 45 nefrectomias utilizando a técnica vídeo-assistida, com ligadura dos vasos renais com clips de titânio (LT-300) e fio cirúrgico. As variáveis analisadas foram tempo cirúrgico, perda sangüínea, tempo de isquemia quente, permanência hospitalar, necessidade de conversão e complicações. Resultados: O procedimento foi realizado com sucesso em todos os casos. O tempo cirúrgico médio foi de 118 minutos, com perda sangüínea estimada em $84 \mathrm{ml}$ e tempo de isquemia quente de 4,3 minutos. Dois casos de íleo prolongado, uma lesão de veia gonadal, um escape de artéria renal e uma necrose de ureter foram observados. A permanência hospitalar média foi de 3,7 dias. O uso de clips vasculares e fio cirúrgico reduziu a perda de tecido venoso comparado à técnica com staplers e gerou redução de custos. Conclusões: A nefrectomia vídeo-assistida com a técnica descrita é factível e mostrou ser efetiva na contenção de gastos e na redução de tecido venoso perdido (Rev. Col. Bras. Cir. 2005; 32(1): 36-40).
\end{abstract}

Descritores: Transplante de rim; Transplante/métodos; Laparoscopia; Doadores vivos; Nefrectomia.

\section{INTRODUÇÃO}

O primeiro caso de nefrectomia laparoscópica em doador vivo foi relatado por Ratner et al ${ }^{1}$ em 1995 e desde então a técnica laparoscópica vem sendo aceita como uma via de acesso alternativa à cirurgia tradicional a céu aberto, com as vantagens de apresentar menor perda sangüínea, melhor efeito estético final da cicatriz, menor dor pós-operatória, permanência hospitalar diminuída e retorno ao trabalho preco$\mathrm{ce}^{2-14}$. Os resultados a curto e a longo prazo com relação à função do enxerto são equivalentes à nefrectomia a céu aberto ${ }^{3}$. No entanto, apesar do sucesso da nefrectomia laparoscópica em doadores vivos, a curva de aprendizado associada a esta técnica permanece um desafio para a maioria dos cirurgiões.

A cirurgia laparoscópica vídeo-assistida é uma técnica minimamente invasiva desenvolvida para facilitar a realização das nefrectomias ${ }^{15} \mathrm{e}$ utiliza uma incisão abdominal (necessária para a retirada do órgão íntegro da cavidade abdominal) através da qual é introduzida a mão do cirurgião ou do auxiliar. Esta técnica facilita a dissecção do pólo renal superior e do ureter ${ }^{16}$, reduz a curva de aprendizado, diminui o tempo de cirurgia e de isquemia quente $\mathrm{e}^{5,17,18}$, com resultados semelhantes à técnica laparoscópica convencional ${ }^{19}$.
Durante os últimos três anos a nefrectomia vídeoassistida se mostrou a técnica mais adequada aos pacientes e à equipe cirúrgica do nosso serviço, e nenhuma nefrectomia a céu aberto foi realizada após o início do programa de laparoscopia para os doadores de transplante renal. A maioria dos cirurgiões utiliza staplers para a ligadura da veia e artéria renais, mas a nossa equipe cirúrgica optou por realizar a ligadura dos vasos renais usando clips vasculares e fio cirúrgico para reduzir custos e para diminuir a perda de tecido vascular. O objetivo do presente artigo é relatar a experiência do nosso serviço usando a técnica descrita nas cirurgias vídeo-assistidas em doadores renais vivos.

\section{MÉTODO}

Todos os doadores renais em potencial que se apresentaram ao nosso serviço, de fevereiro de 2001 a julho de 2003, foram considerados candidatos à nefrectomia vídeoassistida. Cada doador foi submetido à avaliação médica e imunológica pré-operatória para confirmar a possibilidade de doação. Os exames requisitados para delinear a anatomia vascular renal no pré-operatório foram aqueles solicitados de rotina para doadores renais convencionais, incluindo angiografia e urografia excretora.

1. Cirurgião Geral do Hospital da Cruz Vermelha.

2. Cirurgião Geral e Urologista do Hospital da Cruz Vermelha.

3. Residente de Cirurgia Geral do Hospital Universitário Cajuru e da Irmandade Santa Casa de Misericórdia de Curitiba (Aliança Saúde - PUC-PR).

4. Cirurgião Geral do Hospital Municipal de São José.

5. Nefrologista do Hospital da Cruz Vermelha.

6. Acadêmica de Medicina da Pontifícia Universidade Católica do Paraná

Trabalho realizado no Hospital Municipal de São José - Joinville - SC e no Hospital da Cruz Vermelha - Curitiba - PR. 


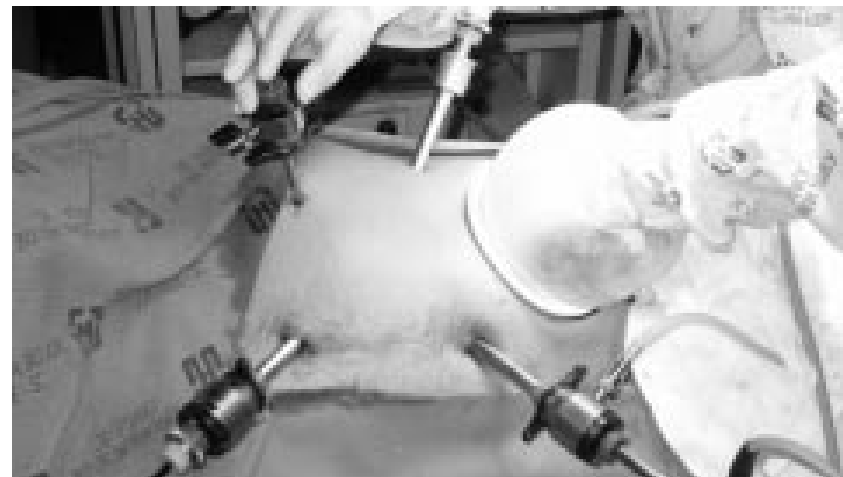

Figura 1 - Posicionamento dos trocáteres e do hand-port no caso de uma nefrectomia esquerda.

A escolha do rim a ser retirado seguiu o princípio básico de preservar o melhor rim in situ para o doador, independentemente do lado e do número de vasos renais.

Durante este período, 45 nefrectomias vídeo-assistidas foram realizadas seguindo a técnica abaixo relatada. As variáveis analisadas foram tempo cirúrgico, perda sangüínea, tempo de isquemia quente, permanência hospitalar, necessidade de conversão e complicações.

\section{Técnica Cirúrgica}

O doador é posicionado em decúbito lateral a $45^{\circ}$, contralateral ao rim a ser doado. Uma incisão de 6 a 8 centímetros é realizada na fossa ilíaca, com abertura da parede abdominal por planos, sem secção muscular. A cavidade abdominal é inspecionada cuidadosamente e uma compressa úmida é introduzida para afastar o cólon e auxiliar em eventual sangramento. Após o afastamento colônico, o ureter é identificado e reparado por um dreno de penrose número 1 . O handport é posicionado e a mão do primeiro auxiliar é introduzida na cavidade abdominal. A câmera é manipulada pelo primeiro auxiliar e o cirurgião mantém as duas mãos livres para manusear as pinças de vídeo-laparoscopia. A cavidade é lentamente insuflada com uma pressão de $14 \mathrm{mmHg}$.

Uma punção de $10 \mathrm{~mm}$ é realizada na região umbilical para a introdução da ótica de $30^{\circ}$; outras duas punções de $10 \mathrm{~mm}$ são realizadas, uma a meia distância entre o apêndice xifóide e o umbigo, e a outra na linha axilar anterior a nível umbilical. Eventualmente um trocáter de $5 \mathrm{~mm}$ é posicionado do lado direito para afastar o fígado ou do lado esquerdo para afastar o baço (Figura 1).

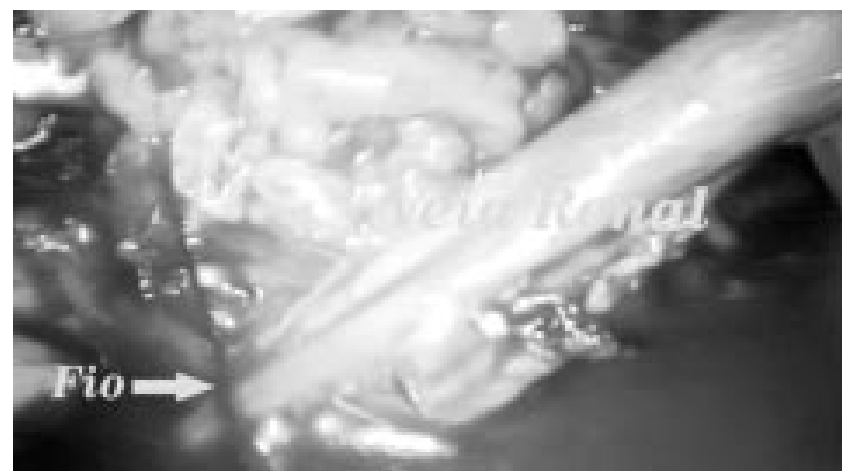

Figura 3 - O nó previamente preparado é amarrado com o intuito de reduzir o calibre da veia para permitir a aplicação dos clips com segurança.

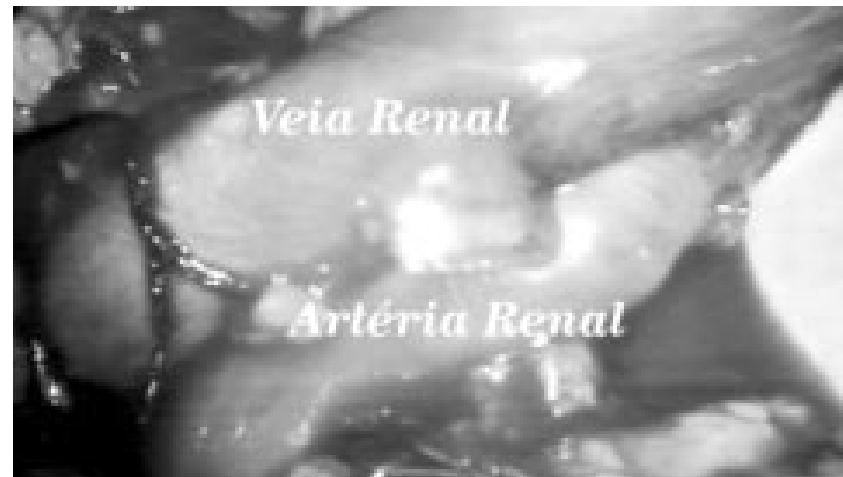

Figura 2 - Passagem do fio de algodão número 0 ao redor da veia renal e preparação do nó.

A dissecção se inicia pela liberação do pólo renal inferior, porção renal posterior e pólo renal superior. Essa dissecção pode ser realizada mais facilmente uma vez que a presença da mão auxiliar facilita o controle do rim e evita a rotação ou potenciais danos ao hilo renal. $\mathrm{O}$ ureter, reparado pelo dreno de penrose, é dissecado cranial e caudalmentemente até o ponto onde cruza os vasos ilíacos, cuidando para que o tecido periureteral (entre o pólo renal inferior e o ureter) seja mantido intacto para evitar a desvascularização do ureter. O pedículo renal é dissecado amplamente e liberado das estruturas adjacentes. As colaterais da veia renal, quando presentes, são ligadas com clips de titânio. É imperativo limpar completamente todo o tecido adiposo e linfático ao redor da artéria e da veia renais para que os clips hemostáticos possam ser posicionados adequadamente, sem risco de deslocamento. A artéria e a veia renais são dissecadas até o nível da aorta e da veia cava inferior, respectivamente.

Após o completo isolamento dos vasos renais, o rim é tracionado lateralmente com a mão, estirando-se os vasos renais. Um fio inabsorvível de algodão número 0 é passado ao redor da veia renal laparoscopicamente e é levemente ajustado o mais proximal possível à veia cava, deixando o nó pronto para ser apertado após a secção da artéria renal (Figura 2). Dois clips de titânio (LT-300) são aplicados na artéria renal e esta é seccionada. O calibre da veia renal é estreitado amarrando o nó previamente preparado (Figura 3) e dois clips são aplicados na veia renal, imediatamente laterais ao nó (Figura 4). A veia é dividida e o rim é removido através do hand-port, completando a secção do ureter a céu aberto (Figura 5).

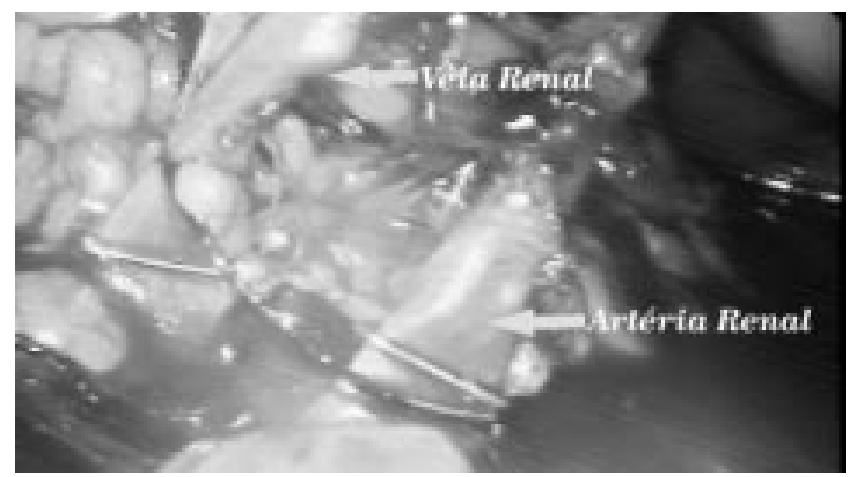

Figura 4 - Aplicação dos clips LT-300 na veia renal, imediatamente laterais ao nó. 


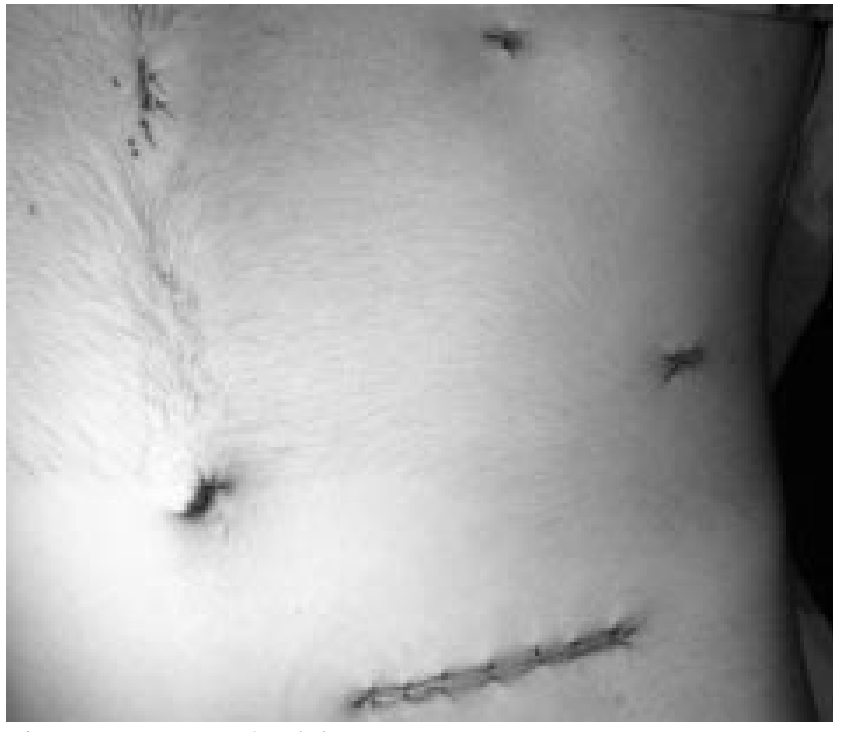

Figura 5 - Aspecto final da cirurgia.

\section{RESULTADOS}

O procedimento foi realizado com sucesso em todos os casos e nenhum paciente necessitou conversão para laparotomia. Foram incluídos 22 homens e 23 mulheres com uma

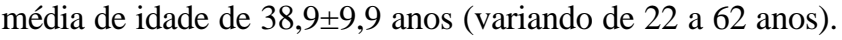
Dezoito doadores foram submetidos à nefrectomia direita e 27 à nefrectomia esquerda. Anatomicamente, trinta e nove rins apresentavam artéria única, cinco possuíam duas artérias e em um caso três artérias estavam presentes. Apenas um caso apresentou duas veias renais; os demais possuíam veia renal única.

O tempo cirúrgico médio em nossa casuística foi de $118,2 \pm 34,9$ minutos (variando de 55 a 190 minutos). A perda sangüínea média estimada foi de $84,1 \pm 79 \mathrm{ml}$ (variando de 10 a $450 \mathrm{ml}$ ) por paciente e nenhum deles necessitou transfusão de hemoderivados. O tempo de isquemia quente, definido como o tempo transcorrido entre a ligadura da artéria renal e a perfusão renal com a solução de preservação, foi de 4,3 $\pm 2,4$ minutos (variando de 1,5 a 11 minutos).

Todos os pacientes receberam dieta no primeiro dia de pós-operatório. As complicações apresentadas nos doadores foram dois casos de íleo prolongado $(4,4 \%)$, uma lesão de veia gonadal $(2,2 \%)$ e um escape de artéria renal $(2,2 \%)$. Ambos os casos de íleo prolongado se resolveram espontaneamente, e os pacientes receberam alta no sétimo dia de pósoperatório. A lesão de veia gonadal foi reparada com sucesso por via laparoscópica e o paciente com escape do clip da artéria renal necessitou de laparotomia de emergência seis horas após o procedimento cirúrgico inicial para controle do sangramento, que foi obtido com sucesso. Um receptor apresentou necrose de ureter $(2,2 \%)$ após a retirada do catéter duplo J e evoluiu bem após o reimplante do mesmo.

O tempo de permanência hospitalar médio dos doadores renais foi de 3,7 $\pm 1,2$ dias (variando de 2 a 7 dias).

\section{DISCUSSÃo}

Apesar do grande sucesso do transplante renal com doadores vivos é de extrema importância reduzir ao máximo a morbidade aos doadores. A doação renal com o procedimento cirúrgico tradicional a céu aberto é associada a considerável morbidade, principalmente relacionada à magnitude da operação e ao tamanho da incisão $0^{2,8-10,19}$. Durante a última década, uma alternativa aos procedimentos abertos tradicionais vem sendo a cirurgia minimamente invasiva ${ }^{20}$, descrita como uma modalidade cirúrgica que usa a técnica laparoscópica clássica, combinada ou não à aplicação da mão do cirurgião como uma ferramenta para auxiliar a dissecção renal (técnica vídeoassistida).

O primeiro caso de nefrectomia laparoscópica em doador vivo foi relatado por Ratner et al ${ }^{1}$ em 1995, que retirou o rim através de uma incisão infraumbilical de $9 \mathrm{~cm}$. Como uma incisão era necessária à retirada do rim após a nefrectomia laparoscópica clássica, parecia lógico tentar utilizar esta incisão para facilitar o procedimento cirúrgico. Assim, três anos mais tarde, Wolf et al ${ }^{15}$ realizaram a primeira nefrectomia vídeoassistida em doador vivo, combinando visualização laparoscópica e facilidade técnica gerada pela assistência manual. Após esses relatos, os procedimentos minimamente invasivos emergiram como alternativas plausíveis à cirurgia a céu aberto em doadores renais para transplante $\mathrm{e}^{3,67,11,21-23}$, e recentemente têm se tornado a via de acesso de escolha em vários programas de transplante renal em todo o mundo ${ }^{14}$.

As vantagens da cirurgia laparoscópica são bastante evidentes e bem relatadas na literatura, e incluem menor desconforto pós-operatório, menor necessidade de analgesia e de permanência hospitalar, melhor resultado estético e recuperação pós-operatória mais rápida, com retorno precoce às atividades diárias e ao trabalho ${ }^{1,2,4-6,8-10,12-14}$. Todos esses benefícios são obtidos com o acesso laparoscópico, com resultados equivalentes à cirurgia aberta com relação ao enxerto renal $^{7,12,24}$. Há uma série de vantagens comparando a cirurgia vídeo-assistida à técnica laparoscópica convencional, resultante de facilidades técnicas como maior sensibilidade tátil e habilidade para o controle e exposição do rim, notadamente do hilo renal ${ }^{18}$. O pólo renal superior é dissecado mais facilmente ${ }^{5}$, o cólon é mobilizado com facilidade, os sangramentos inesperados são controlados mais rapidamente, a exposição dos vasos é otimizada na hora do grampeamento com staplers ou clips de titânio, a torção dos vasos renais é evitada e a chance de lesão esplênica é menor ${ }^{18}$. A técnica vídeo-assistida facilita a ligadura dos vasos renais e a retirada do rim, reduzindo o tempo cirúrgico, o tempo de isquemia quente e a curva de aprendizado ${ }^{5,17,18}$. Vários centros têm documentado um aumento no número de doações nos últimos anos e têm atribuído este aumento ao advento das técnicas minimamente invasivas, que são mais bem aceitas pelos doadores renais ${ }^{4,6,9}$.

Algumas desvantagens relacionadas à cirurgia vídeoassistida comparada à laparoscopia tradicional incluem incisão abdominal 1 a 2 centímetros maior, dependendo do tamanho da mão do cirurgião ou do auxiliar, tempo de recuperação discretamente maior e retorno da função intestinal um pouco mais lenta ${ }^{18}$.

Geralmente o rim esquerdo é preferido para transplante renal de doador vivo devido ao maior comprimento da veia renal. A nefrectomia direita é tecnicamente mais complicada e mais desafiadora, uma vez que é necessário o afastamen- 
to do fígado e a veia renal direita é mais curta do que a esquerda, aumentando a chance de trombose do enxerto ${ }^{25}$. Para evitar esse tipo de complicação, deve-se atentar para detalhes técnicos para obter um comprimento vascular suficiente. A dissecção da artéria e da veia renal deve ser realizada até a origem na aorta e a desembocadura na veia cava, respectivamente ${ }^{26}$. A aplicação de staplers para dividir a veia renal resulta em perda de aproximadamente 1 a $1,5 \mathrm{~cm}$ de comprimento de enxerto vascular devido à linha de grampos ${ }^{27}$, o que pode dificultar a anastomose no receptor. Para reduzir a quantidade de tecido perdida com o grampeamento desenvolveu-se a técnica descrita com fio cirúrgico inabsorvível de algodão número 0 e clips de titânio, acarretando um comprometimento de apenas 4 a $6 \mathrm{~mm}$ de extensão de tecido venoso em nossa casuística. Além disso, enquanto a eficácia das duas técnicas para controle dos vasos renais é semelhante, a utilização de fio cirúrgico e clips de titânio está associada a uma redução nos custos totais do procedimento, economizando cerca de 700 dólares por stapler Endo-GIA.

Apesar da relutância em se utilizar rins direitos para o transplante renal em doadores vivos, nossa casuística engloba 18 casos de nefrectomias direitas e a equipe cirúrgica apresenta uma preferência por cirurgias deste lado, uma vez que a veia renal direita apresenta um menor número de ramos colaterais $^{28}$. Além disso, a realização de nefrectomias direitas nos permite preservar o princípio básico de manter o melhor rim in situ para o doador nos casos em que a função renal esquerda é superior à direita.

Avaliando os resultados obtidos em nossa série e comparando os dados àqueles da literatura internacional, encontramos resultados bastante semelhantes e encorajadores. O tempo cirúrgico médio, desde a incisão inicial até o fechamento da pele, foi de 118,2 $\pm 34,9$ minutos, e está dentro dos valores relatados na literatura internacional (121,2 a 280 minutos $)^{5,22,28,29}$. A perda sangüínea média de $64.3 \pm 56.9 \mathrm{ml}$ encaixa-se nos valores aceitos internacionalmente, de 125 a $275 \mathrm{ml}^{22,28,29}$, e o tempo de isquemia quente de 3.4 \pm 1.7 minutos é comparável a outros estudos ${ }^{5,22,28,29}$, mas um pouco superior à experiência de Ruiz-Deya et $l^{18} \mathrm{e}$ Wolf et al $^{19}$, que relataram 1,8 e 2,9 minutos, respectivamente. No en- tanto, esse tempo de isquemia quente não acarretou nenhum dano ao enxerto renal, e diurese imediata foi estabelecida em $100 \%$ dos nossos receptores. A trombose vascular é evidenciada em até $2,7 \%$ dos receptores ${ }^{27}$, mas nenhum caso ocorreu em nossa casuística. A incidência de complicações ureterais após nefrectomia laparoscópica pode variar de 2 a $11 \%{ }^{21,29}$, e um caso de necrose de ureter $(2,2 \%)$ após a retirada do catéter duplo J foi observado, mas com boa evolução após ser reimplantado. Taxas de conversão para cirurgia aberta de 1,5 a 6\% são relatadas na literatura ${ }^{2,7,8,22,29}$ e felizmente nenhum dos nossos pacientes necessitou conversão cirúrgica. Em um caso houve necessidade de laparotomia 6 horas após o procedimento cirúrgico inicial para nova ligadura da artéria renal, cujos clips de titânio haviam soltado. O sangramento foi controlado e o paciente apresentou excelente curso pós-operatório. Acreditamos que possíveis causas do deslocamento dos clips sejam falha técnica no momento da aplicação dos clips, falha relacionada ao material cirúrgico e mobilização dos clips no momento da retirada da compressa usada para o afastamento do cólon. A causa definitiva, no entanto, permanece obscura.

O tempo de permanência hospitalar médio foi de 3,7 $\pm 1,2$ dias, compatível com a hospitalização média de vários centros de referência (2,2 a 4,4 dias) $)^{2,30}$.

A nefrectomia vídeo-assistida usando fio cirúrgico e clips de titânio para controle dos vasos renais é uma técnica factível e pode oferecer algumas vantagens quando comparada à técnica usando staplers. Um adequado comprimento de enxerto vascular é obtido, reduzindo a perda de tecido venoso para 4 a $6 \mathrm{~mm}$, ao invés de 10 a $15 \mathrm{~mm}$ com staplers Endo-GIA. Uma redução dos custos é alcançada utilizando esta técnica, fator importante para permitir a realização desse tipo de procedimento minimamente invasivo em nosso meio.

\section{AGRADECIMENTOS:}

\author{
Marco Aurélio de George, Cirurgião Geral do Hospital da Cruz \\ Vermelha e \\ Marlon Rangel,ACBC-PR, Cirurgião Geral do Hospital da Cruz \\ Vermelha pela contribuição na realização deste trabalho.
}

\begin{abstract}
Background: Laparoscopic live donor nephrectomy has acquired an important role in the minimally invasive surgery era, decreasing morbidity to kidney donors, with an equivalent renal graft outcome compared with open surgery. The aim of this article is report our experience using the technique of renal vessels control with metallic clips and cotton suture. Methods: Fourty-five nephrectomies were performed following the hand-assisted technique and using titanium clips (LT-300) and cotton suture for renal vessels ligatures. Operative data and postoperative courses were reviewed, including surgical time, estimated blood loss, warm ischemia time, length of hospital stay, conversion to laparotomy, and complications. Results: The procedure was performed successfully in all cases, including 18 right nephrectomies and 27 left nephrectomies. The mean operative time in our series was 118 minutes, with an estimated blood loss of $84 \mathrm{ml}$ and warm ischemia time of 4.3 minutes. Two cases of postoperative ileus, one gonadal vein lesion, one metallic clip displacement and one ureteral necrosis were observed. The mean postoperative hospital stay was 3.7 days. The use of titanium clips and cotton suture reduced the loss of venous tissue compared to the technique using the Endo-GIA stapling device (4 to $6 \mathrm{~mm} v \mathrm{~s}$. 10 to $15 \mathrm{~mm}$ ) and showed to be associated with a cost reduction, saving about 700 US dollars per stapler. Conclusions: Hand-assisted nephrectomy using the above described technique is technically feasible and showed to be effective in reducing costs and decreasing the loss of vascular length.
\end{abstract}

Key words: Kidney transplantation; Transplantation/methods; Laparoscopy; Living donors; Nephrectomy. 


\section{REFERÊNCIAS}

1. Ratner LE, Ciseck LJ, Moore RG, et al. - Laparoscopic live donor nephrectomy. Transplantation,1995,60(9):1047-1049.

2. Flowers JL, Jacobs S, Cho E, et al. - Comparison of open and laparoscopic live donor nephrectomy. Ann Surg,1997,226(4): 483-490.

3. Fabrizio MD, Ratner LE, Montgomery RA, et al. - Laparoscopic live donor nephrectomy. Urol Clin North Am,1999,26(1):247256.

4. Odland MD, Ney AL, Jacobs DM, et al. - Initial experience with laparoscopic live donor nephrectomy. Surgery,1999,126(4): 603-607.

5. Slakey DP, Wood JC, Hender D, et al. - Laparoscopic living donor nephrectomy: advantages of the hand-assisted method. Transplantation, 1999,68(4):581-583.

6. Ratner LE, Montgomery RA, Kavoussi LR - Laparoscopic live donor nephrectomy: the four year Johns Hopkins University experience. Nephrol Dial Transplant,1999, 14(9):2090-2093.

7. Jacobs SC, Cho E, Dunkin BJ, et al. - Laparoscopic living donor nephrectomy: the university of Maryland 3-year experience. J Urol,2000,164(5):1494-1499.

8. Kuo PC, Johnson LB, Sitzmann JV - Laparoscopic donor nephrectomy with a 23-hour stay: a new standard for transplantation surgery. Ann Surg,2000,231(5):772-779.

9. Kuo PC, Johnson LB - Laparoscopic donor nephrectomy increases the supply of living donor kidneys. Transplantation,2000,69(10):2211-2213.

10. Schweitzer EJ, Wilson J, Jacobs S, et al. - Increased rates of donation with laparoscopic donor nephrectomy. Ann Surg,2000,232(3):392-400.

11. Montgomery RA, Kavoussi LR, Su L, et al. - Improved recipient results after 5 years of performing laparoscopic donor nephrectomy. Transplant Proc,2001,33(1-2): 1108-1110.

12. Hsu TH, Su LM, Ratner LE, et al. - Laparoscopic donor nephrectomy in the elderly patient. Urology,2002,60(3):398401.

13. Siqueira TM Jr, Gardner TA, Kuo RL, et al. - One versus two proficient laparoscopic surgeons for laparoscopic live donor nephrectomy. Urology,2002,60(3): 406-409.

14. Buell JF, Hanaway MJ, Woodle ES - Maximizing renal artery length in right laparoscopic donor nephrectomy by retrocaval exposure of the aortorenal junction. Transplantation,2003,75(1): 83-85.

15. Wolf JS Jr, Tchetgen MB, Merion RM - Hand-assisted laparoscopic living donor nephrectomy. Urology,1998,52(5): 885-887.

16. Potter SR, Buell JF, Hanaway M, et al. - Laparoscopic live donor nephrectomy: rationale, techniques, and implications. Semin Dial,2001,14(5):365-372.
17. Stifelman MD, Hull D, Sosa RE, et al. - Hand assisted laparoscopic nephrectomy: a comparison with the open approach. J Urol,2001,166(2):444-448.

18. Ruiz-Deya G, Cheng S, Palmer E, et al. - Open donor, laparoscopic donor and hand assisted laparoscopic donor nephrectomy: a comparison of outcomes. J Urol, 2001,166(4):1270-1274.

19. Wolf JS Jr, Marcovich R, Merion RM, et al. - Prospective, case matched comparison of hand assisted laparoscopic and open surgical live donor nephrectomy. J Urol,2000,163(6):1650-1653.

20. Ratner LE, Buell JF, Kuo PC - Laparoscopic donor nephrectomy: pro. Transplantation,2000,70(10):1544-1546.

21. Brown SL, Biehl TR, Rawlins MC, et al. - Laparoscopic living donor nephrectomy: a comparison with the conventional open approach. J Urol, 2001,165(3):766-769.

22. Hawasli A, Boutt A, Cousins G, et al. - Laparoscopic versus conventional live donor nephrectomy: experience in a community transplant program. Am Surg,2001, 67(4):342-345.

23. Velidedeoglu E, Williams N, Brayman KL, et al. - Comparison of open, laparoscopic, and hand-assisted approaches to livedonor nephrectomy. Transplantation, 2002,74(2):169-172.

24. Ratner LE, Hiller J, Sroka M, et al. - Laparoscopic live donor nephrectomy removes disincentives to live donation. Transplant Proc, 1997,29(8):3402-3403.

25. Mandal AK, Cohen C, Montgomery RA, et al. - Should the indications for laparascopic live donor nephrectomy of the right kidney be the same as for the open procedure? Anomalous left renal vasculature is not a contraindiction to laparoscopic left donor nephrectomy. Transplantation,2001,71(5):660-664.

26. Ratner LE, Kavoussi LR, Chavin KD, et al. - Laparoscopic live donor nephrectomy: technical considerations and allograft vascular length. Transplantation, 1998,65(12):1657-1658.

27. Ratner LE, Montgomery RA, Maley WR, et al. - Laparoscopic live donor nephrectomy: the recipient. Transplantation, 2000,69(11):2319-2323.

28. Lind MY, Hazebroek EJ, Hop WC, et al. - Right-sided laparoscopic live-donor nephrectomy: is reluctance still justified? Transplantation,2002,74(7):1045-1048.

29. Leventhal JR, Deeik RK, Joehl RJ, et al. - Laparoscopic live donor nephrectomy - is it safe? Transplantation,2000,70(4): 602-606.

30. Ratner LE, Kavoussi LR, Schulam PG, et al. - Comparison of laparoscopic live donor nephrectomy versus the standard open approach. Transplant Proc,1997,29(1-2):138-139.

Endereço para correspondência:

Dr. Anibal Wood Branco

Rua das Palmeiras, 170 ap. 201

80620-210 - Curitiba - PR - Brasil

Telefone (41) 242-6543

E-mail: anibal@awbranco.com.br 